Sportwiss 2009 · 39:92-92

DOI 10.1007/s12662-009-0062-y

(C) Springer Medizin Verlag 2009

\title{
Online-Einreichung von Manuskripten für Sportwissenschaft
}

- Ihre Daten sind sicher: Sie werden auf dem zentralen Datenserver gespeichert, der regelmäßig gesichert wird, sodass ein Datenverlust nicht vorkommt.

- Mehr Transparenz: Autoren können jederzeit online den aktuellen Stand der Begutachtung ihres eingereichten Artikels verfolgen.

Weitere Informationen zum Verfassen und Einreichen von Manuskripten finden Sie in der nebenstehenden Infobox.

Ihre Schriftleitung Sportwissenschaft

\section{Für die Schriftleitung}

Prof. Dr. Michael Krüger Universität Münster Institut für Sportwissenschaft Horstmarer Landweg 62b 48149 Münster

mkrueger@uni-muenster.de

\section{Manuskripte online einreichen}

Bitte folgen Sie auf der Homepage www.Sportwissenschaft.springer.de dem Link „Für Autoren“.

Klicken Sie bitte unter „Editorial Manager" auf "Manuskript online einreichen“. Hier finden Sie auch die Autorenleitfäden und Musterbeiträge mit Hinweisen für die Manuskriptgestaltung.

Bei der ersten Nutzung registrieren Sie sich unter "Register" mit der Angabe Ihrer persönlichen Daten und eines User-Namen. Ein Passwort wird Ihnen nach Registrierung per E-mail automatisch zugeschickt. Mit Ihrem User-Namen und Passwort haben Sie als Autor zukünftig direkten Zugang zum System. Nach der Anmeldung (Login) als Autor können Sie nun Ihren Beitrag online einreichen.

Sollten Sie noch Fragen zur Manuskripteinreichung haben, wenden Sie sich bitte an die Redaktion beim Herausgeber:

\section{Anna-Elena Gerhards}

Redaktion Editorial Manager der Zeitschrift Sportwissenschaft

Tel. 0251/83-32356

agerhards@uni-muenster.de

www.Sportwissenschaft.springer.de oder direkt zu

www.editorialmanager.com/spwi der Urdatei in eine PDF-Datei - ein stabiles Dokument, das überall an jedem Computer gleich aussieht.

- Der Begutachtungsprozess wird beschleunigt: Das Redaktionssystem ermöglicht die nahtlose Verbindung zwischen Einreichung, Begutachtung, eventueller Revision und schließlich Veröffentlichung. 